\title{
Extraskeletal Ewing's sarcoma: outcomes and CT features of endoceliac lesions
}

\author{
Zeyang Chen ${ }^{1 \#}$, Yang Jiao ${ }^{2 \#}$, Zining Liu ${ }^{3 \#}$, Jiejin Yang ${ }^{4}$, Jiali Sun ${ }^{4}$, Pengyuan Wang ${ }^{1}$ \\ ${ }^{1}$ Department of General Surgery, Peking University First Hospital, Beijing, China; ${ }^{2}$ Department of Orthopaedic, Peking University First Hospital, \\ Beijing, China; ${ }^{3}$ Key laboratory of Carcinogenesis and Translational Research (Ministry of Education/Beijing), Gastrointestinal Cancer Center, \\ Peking University Cancer Hospital \& Institute, Beijing, China; ${ }^{4}$ Department of Radiology, Peking University First Hospital, Beijing, China \\ Contributions: (I) Conception and design: Z Chen, Y Jiao, Z Liu, P Wang; (II) Administrative support: P Wang; (III) Provision of study materials or \\ patients: All authors; (IV) Collection and assembly of data: Z Chen, Y Jiao, J Yang, J Sun; (V) Data analysis and interpretation: Z Chen, Y Jiao, Z Liu, \\ P Wang; (VI) Manuscript writing: All authors; (VII) Final approval of manuscript: All authors. \\ \#These authors contributed equally to this work. \\ Correspondence to: Pengyuan Wang. Department of General Surgery, Peking University First Hospital, Peking University, 8 Xi ShiKu Street, Beijing \\ 100034, China. Email: wangpengyuanpku@163.com.
}

Background: Extraskeletal Ewing's sarcoma (EES) is one kind of rare malignant tumour which is always misdiagnosed preoperatively, especially for lesions located at endoceliac sites. This study analyse the clinicopathological features and outcomes of EES patients. The basic imaging characteristics of endoceliac lesions are also summarized.

Methods: This study involved EES patients admitted to our centre between January 2000 and January 2020. Clinical data from patients with EES (n=25) and computed tomography (CT) features from endoceliac EES patients with available CT data $(n=8)$ were retrospectively reviewed.

Results: The sample comprised 18 males and 7 females with a median age of 30 years (range, 1-72 years). Seven patients had EES originating from surface sites and 18 had EES originating from endoceliac sites. The median tumour size was $8.0 \mathrm{~cm}$ (range, $2.5-17.0 \mathrm{~cm}$ ). In total, $20 \%$ of patients had distant metastasis at diagnosis. In the univariate analyses, tumour size $>8 \mathrm{~cm}$, non-surgical treatment, and regional lymph nodes metastasis were risk factors for poor prognosis of EES. In the multivariate analysis, patients with larger tumour size and regional lymph node metastasis were independent predictors of overall survival (OS). Endoceliac EES cases frequently exhibited lobulated contour (87.5\%), absence of calcification (75\%), severe necrosis or cystic degeneration (75\%), heterogeneous enhancement (100\%), moderate enhancement (75\%), ill-defined borderline (62.5\%), and organ invasion (75\%). Half of the patients with endoceliac EES had CT features of lymphadenopathy.

Conclusions: Comprehensive understanding of the clinicopathological and imaging features of EES is beneficial for diagnosis, development of therapeutic strategies, and accurate prediction of prognosis for this rare malignant tumour.

Keywords: Extraskeletal Ewing's sarcoma (EES); outcomes; computed tomography (CT); endoceliac lesions

Submitted Apr 07, 2021. Accepted for publication Jul 14, 2021.

doi: $10.21037 /$ tcr-21-607

View this article at: https://dx.doi.org/10.21037/tcr-21-607

\section{Introduction}

Extraskeletal Ewing's sarcoma (EES) is a member of the Ewing's sarcoma family of tumours (ESFTs). The ESFTs includes various types of lesions, such as EES, skeletal Ewing's sarcoma (SES), peripheral primitive neuroectodermal tumour (pPNET), and Askin tumour $(1,2)$. Members of the ESFTs have the same morphologic features, ultrastructure, immunophenotypes, cytogenetics, 
and tumour gene expression $(3,4)$. Given that Ewing's sarcoma (EWS) and pPNET have the same neoplastic processes, the World Health Organization has recognized EWS/pPNET as the preferred terminology for soft tissue and bone tumours; terms such as "Askin tumour" are no longer used $(5,6)$.

The skeleton is the most common site for EWS, and EES only accounts for about $20 \%$ of all ESFTs $(7,8)$. As such, there exists a significant quantity of studies focused on the diagnosis and prognosis of SES, such that currently, the treatment strategies for SES are relatively mature (9). However, due to the rarity of EES, there is still a lack of high-quality research of EES and its clinical characteristics, therapeutic strategies, and prognostic factors vary among studies (1,10-12). EES can originate from any site in the body (12); thus, in the current study, patients presented to a wide range of departments, not just the department of bone oncology. Therefore, standard application of appropriate diagnostic and therapeutic strategies is crucial for specialists from various departments (13), such as preoperative biopsy and neoadjuvant chemotherapy. Preoperative diagnosis and thorough imaging of the characteristics of EES are important, especially for endoceliac tumours which are hard to identify by physical examination and are always misdiagnosed preoperatively.

Here, we report and compare the clinical characteristics and outcomes of a series of EES patients at our institution. The aim was to identify significant risk factors for EES prognosis. We also aimed to describe the imaging characteristics of endoceliac EES by reviewing the data from computed tomography (CT). These findings are important for accurate diagnosis and treatment of EES. We present the following article in accordance with the STROBE reporting checklist (available at https://dx.doi. org/10.21037/tcr-21-607).

\section{Methods}

\section{Study subjects}

The study was conducted in accordance with the Declaration of Helsinki (as revised in 2013). The retrospective study was approved by the Institutional Ethics Committee of Peking University First Hospital (No. 2021365 ) and the verbal informed consent was obtained from all the patients. In total, 25 patients with primary EES, who were admitted to our centre from January 2000 to January 2020, were identified in the institution's clinicopathological database. The inclusion criteria were as follows: (I) primary EES and the oncological diagnosis confirmed by two experienced pathologists specialising in sarcoma; (II) patient has detailed clinicopathological data; (III) information on overall survival (OS) of patients is available in the followup data. The extraskeletal origin of each patient was confirmed by imaging and pathological examinations, with findings indicating no bone marrow involvement; however, patients with oppression and reactive changes in the cortex of the bone adjacent to the EES were not excluded from our study. For the pathological examination, after excluding other small round cell tumours, such as lymphoma, rhabdomyosarcoma, and neuroblastoma, the still inconclusive cases underwent fluorescent in situ hybridization (FISH) to confirm the presence of chromosomal translocation involving the EWSR1 gene $[\mathrm{t}(11 ; 22)(\mathrm{q} 24 ; \mathrm{q} 12)]$.

\section{Treatment}

All patients who underwent surgery or had unresectable disease received chemotherapy as an adjuvant or maintenance therapy. The chemotherapy protocols varied among the patients due to the time span of our study. Eight patients were treated with vincristine $2 \mathrm{mg} / \mathrm{m}^{2}$, doxorubicin $25-75 \mathrm{mg} / \mathrm{m}^{2}$, cyclophosphamide $900-1,200 \mathrm{mg} / \mathrm{m}^{2}$ (VAC) alternating with etoposide $100 \mathrm{mg} / \mathrm{m}^{2}$ and ifosfamide $1,800 \mathrm{mg} / \mathrm{m}^{2}$ (IE) (1). The remaining patients received multiagent chemotherapy protocols based on vincristine, doxorubicin, cyclophosphamide, etoposide, ifosfamide, and cisplatinum. Only three patients received core needle biopsy and neoadjuvant chemotherapy before surgery. Seventeen patients underwent resection and postoperative chemotherapy. In addition, five patients obtained the histological diagnosis by core needle biopsy and received non-surgical treatment because of unresectable lesions, including metastatic diseases or lesions involving important body parts, such as the facial region. Only two patients underwent postoperative radiotherapy because of suboptimal margins.

\section{Data collection}

The demographic and outcome data included age, gender, primary tumour site, tumour size, distant metastasis, site of metastasis, therapeutic strategies, status of margins, regional lymph node metastasis, and OS. The tumour site was classified as either a surface or endoceliac tumour 
according to the location of the lesion: surface tumours included those on the extremities, facial region, chest wall, and inguinal region; endoceliac tumours included those located in the retroperitoneum, prostate, adrenal gland, kidney, ovary, stomach, and lungs. The tumour size, i.e., the largest diameter, was measured based on imaging examinations, including CT, magnetic resonance imaging (MRI), and ultrasound, before the patient began treatment. The metastatic status was evaluated based on bone scan, chest CT scan, bone marrow biopsy, or positron emission tomography scan. Margins and regional lymph node metastasis were confirmed by pathological examination. The OS was calculated from the date of diagnosis to the date of last follow-up or death.

\section{CT imaging analysis}

Contrast-enhanced CT images from endoceliac EES patients were reviewed by two experienced radiologists who were blind to the clinicopathological data of the patient. The two radiologists reached an agreement by negotiating when there were divergences in opinion. Eight patients with endoceliac EES had complete contrast-enhanced CT imaging data available for review in our institution; CT features included contour, calcification, necrosis or cystic degeneration, degree of enhancement, enhancement pattern, lymphadenopathy, borderline, and organ invasion were reviewed. The contour was classified as round or lobulated according to the tumour's regular or irregular shape. The presence of high-attenuation foci in the tumour was defined as calcification. When the CT value of the object region was less than 20 Housefield units (HUs) in the portal phase, the tumour was classified as exhibiting necrosis or cystic degeneration, the degree of which was graded as follows: mild, $<50 \%$ low-density area in the lesion, and severe, $>50 \%$ low-density area in the lesion. The degree of enhancement was determined by comparing the CT value of the lesion to the muscle and liver in the portal phase, as described previously (14). The enhancement pattern was classified as either heterogeneous or homogeneous. Lymphadenopathy was recorded when a regional lymph node had a short diameter $>1.5 \mathrm{~cm}$ (6). An ill-defined borderline was recorded when the lesion involved the adjacent tissue and had a blurred margin.

\section{Statistical analysis}

The associations between clinical and pathological factors and long-term OS were assessed using the Kaplan-Meier method (log-rank test). Cox proportional-hazards models were used to determine hazard ratios (HR) in univariate and multivariate analyses. Tumour or treatment characteristics for which univariate analysis reached values of $\mathrm{P}<0.05$ were tested in multivariate models. To achieve an exact statistical analysis, the rule of thumb is 10 events per variable in the Cox regression. For all analyses, $\mathrm{P}<0.05$ was considered statistically significant.

Statistical analyses were performed using SE STATA (Stata Statistical Software, release 15.1; Stata Corp., College Station, TX, USA).

\section{Results}

\section{Patient characteristics}

Over the 20-year study period, 25 patients with primary EES were treated at Peking University First Hospital and were eligible for inclusion in this study. The patient characteristics are shown in Table 1. The sample comprised 7 females and 18 males with a male:female ratio of 2.6:1. The median age of the sample was 29 years (range, 1-72 years). The median tumour size was $8 \mathrm{~cm}$ (range, $3.5-17.0 \mathrm{~cm})$. Seven patients $(28 \%)$ presented with surface lesions and $18(72 \%)$ had lesions located at endoceliac sites. Overall, $80 \%$ of patients had localised disease at diagnosis and $20 \%$ had metastatic disease. The lung was the most common metastatic sites for EES in our study.

\section{Outcome analysis}

The median OS was 22 months (range, 2-139 months). Results of the univariate analyses are presented in Table 2. According to the log-rank test, age, gender, suboptimal margins, and neoadjuvant chemotherapy were not prognostic predictors of OS (all $\mathrm{P}>0.05$ ). There was also no significant difference in OS between localised and metastatic disease (HR 2.06, 95\% CI: 0.72-5.87, $\mathrm{P}=0.15$ ). Surface tumours, rather than endoceliac tumours, were associated with improved OS; though, this was not statistically significant (HR 2.51, 95\% CI: 0.78-7.51, $\mathrm{P}=0.101)$. Significant benefits in terms of OS were observed in patients who underwent surgery (HR 5.20, 95\% CI: 1.45-18.62, $\mathrm{P}=0.003$ ) and those with smaller tumour sizes (HR 2.48, 95\% CI: 0.95-6.44, $\mathrm{P}=0.045$ ). Among those who received surgery, patients without regional lymph node metastasis had significantly increased OS (HR 3.94, 
Table 1 Demographic and clinicopathologic characteristics of 25 eligible patients

\begin{tabular}{|c|c|c|}
\hline Characteristics & Values & $\%$ \\
\hline Age, median [range] & $30[1-72]$ & NA \\
\hline \multicolumn{3}{|l|}{ Gender } \\
\hline Female & 7 & 28.0 \\
\hline Male & 18 & 72.0 \\
\hline \multicolumn{3}{|l|}{ Primary tumour site } \\
\hline Surface & 7 & 28.0 \\
\hline Extremity & 1 & 4.0 \\
\hline Facial region & 2 & 8.0 \\
\hline Chest wall & 3 & 12.0 \\
\hline Inguinal region & 1 & 4.0 \\
\hline Endoceliac & 18 & 72.0 \\
\hline Retroperitoneal & 4 & 16.0 \\
\hline Prostate & 3 & 12.0 \\
\hline Kidney & 6 & 24.0 \\
\hline Adrenal gland & 1 & 4.0 \\
\hline Pelvis & 1 & 4.0 \\
\hline Ovary & 1 & 4.0 \\
\hline Stomach & 1 & 4.0 \\
\hline Lung & 1 & 4.0 \\
\hline $\begin{array}{l}\text { Tumour size }(\mathrm{cm}), \text { median } \\
\text { [range] }\end{array}$ & $8[3.5-17.0]$ & NA \\
\hline \multicolumn{3}{|l|}{ Distant metastasis } \\
\hline No & 20 & 80.0 \\
\hline Yes & 5 & 20.0 \\
\hline \multicolumn{3}{|l|}{ Site of metastasis $(n=5)$} \\
\hline Lung & 2 & 40.0 \\
\hline Liver & 1 & 20.0 \\
\hline Peritoneum & 1 & 20.0 \\
\hline Bone & 1 & 20.0 \\
\hline
\end{tabular}

95\% CI: 1.10-14.09, P=0.021). Kaplan-Meier curves for the relationships between OS and tumour size, primary tumour site, distant metastasis, surgery, and regional lymph node metastasis are presented in Figure 1. A Cox proportional hazards model was constructed for the subsample of patients who underwent surgical treatment $(n=20)$; this model was adjusted for potential confounders. The included variables, based on the log-rank tests with $\mathrm{P}<0.05$, were regional lymph nodes metastasis and tumour size (Table 3). The results revealed that regional lymph node metastasis was an independent prognostic factor associated with OS (HR 3.72, 95\% CI: $1.02-13.61, \mathrm{P}=0.047$ ).

\section{CT imaging findings}

Only 8 patients with endoceliac EES have available CT data in our center and their CT features are summarised in Figure 2. Among these patients, 87.5\% (7/8) presented with a lobulated contour, while calcification was detected in only $25 \%$ of the cases $(2 / 8)$. Six patients $(6 / 8 ; 75 \%)$ had severe necrosis or cystic degeneration and all cases presented with heterogeneous enhancement. In total, $75 \%$ of the patients (6/8) had a moderate degree of enhancement and the remaining two patients (25\%) showed a low degree of enhancement. Half of the patients $(50 \%)$ presented with lymphadenopathy and five patients $(5 / 8,62.5 \%)$ had an illdefined borderline. Organ invasion was observed in $75 \%$ of the patients (6/8). Some typical CT images of endoceliac EES patients were shown in Figures 3-5.

\section{Discussion}

The ESFTs are a group of highly malignant diseases with short clinical courses and poor prognosis; these tumours generally present with early metastasis. Although SES is the third most common connective tissue malignancy in children (5), we still have little knowledge of the biological behaviour and prognosis of EES. Notably, to our knowledge, this study reports the largest series of Chinese EES cases to date.

Consistent with the available literature, the male preponderance of EES was also observed in our sample $(10,15-17)$. Researches report that SES patients are younger than EES patients $(1,10,13)$. In the current study, our EES patients were all around 30 years of age and there were no significant differences in OS between subgroups of different ages. Research also indicates that EES can originate from anywhere in the body, but the most common site for EES is the deep soft tissue of extremities (18). However, patients with surface lesions, including tumours located in extremities, only made up a small percentage of the patients with EES in our study. This may be because the general and urinary departments are the specialty departments of our institution, and thus, patients with EES originating from 
Table 2 Univariate regression of overall survival and $\mathrm{P}$ values for $\log$-rank test

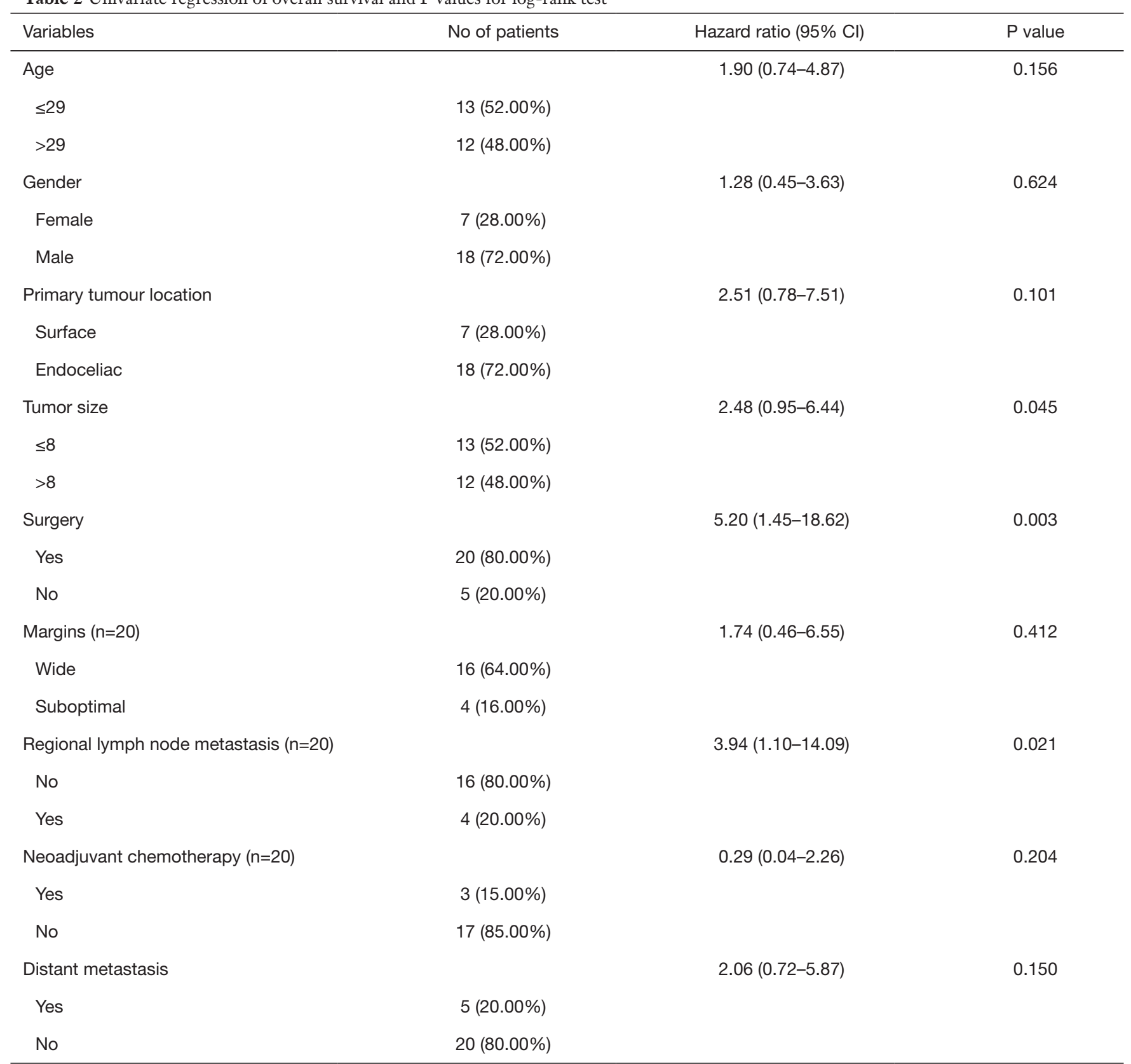

Univariate Cox regression used to determine hazard ratio; log-rank p-values reported; Cl, confidence interval.

the gastrointestinal tract and urinary system were more commonly admitted to our institution. Javery et al. reported that abdominal EES commonly originates from the kidneys or retroperitoneum (7). Similarly, the most common sites of endoceliac EES in our research were the kidneys and retroperitoneum. Omori et al. reported that EES arising in cutaneous or subcutaneous tissue was associated with favourable prognosis compared to deep EES (19). Further, the prognosis of patients with EES arising from the abdomen is reported to be poorer than that of patients with EES arising from other sites (20). The abundant blood circulation and more common organ invasion may lead to the poorer prognosis of deep EES. Although the difference was not statistically significant, in our study we found that endoceliac EES tended to be associated with decreased OS as compared to surface EES. Further, in accordance 

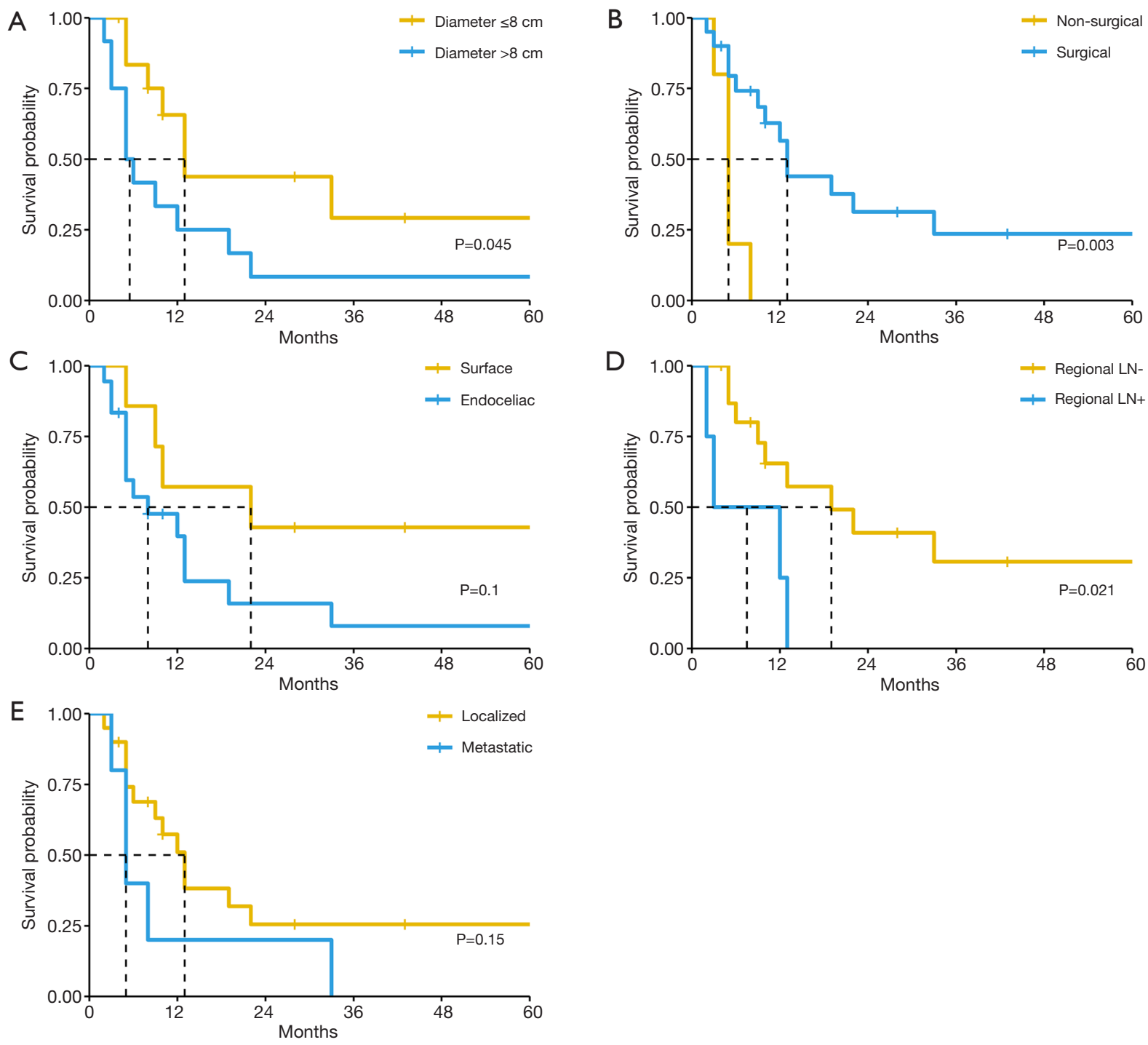

Figure 1 Kaplan-Meier curves of overall survival according to the tumor short axis (A), treatment (B), location (C), lymph nodes positivity (D), evidence of metastasis (E).

Table 3 Multivariate analysis for OS in patients underwent surgery using a Cox proportional hazards model

\begin{tabular}{lcc}
\hline Variables & Hazard ratio $(95 \% \mathrm{Cl})$ & P value \\
\hline Tumour size & $2.56(0.82-8.00)$ & 0.106 \\
$\leq 8$ & & \\
$>8$ & & \\
Regional lymph node & $3.72(1.02-13.61)$ & 0.047 \\
metastasis & & \\
No & & \\
Yes & \\
\hline
\end{tabular}

$\mathrm{Cl}$, confidence interval. with previous studies $(1,21)$, the present study found that increased tumour size, which was associated with later local staging, was a risk factor for poor EES prognosis.

Distant metastasis has been confirmed to be an independent risk factor for poor EES prognosis in several studies $(10,11,13,22)$. Contrary to expectations, this study did not find a significant difference in OS between localised and metastatic disease. The lack of significant difference might be due to the limitation resulting from small group and nonstandard treatment strategy in our center. In a previous study, effective systemic chemotherapy was associated with a 5-year survival rate of localised EES in 


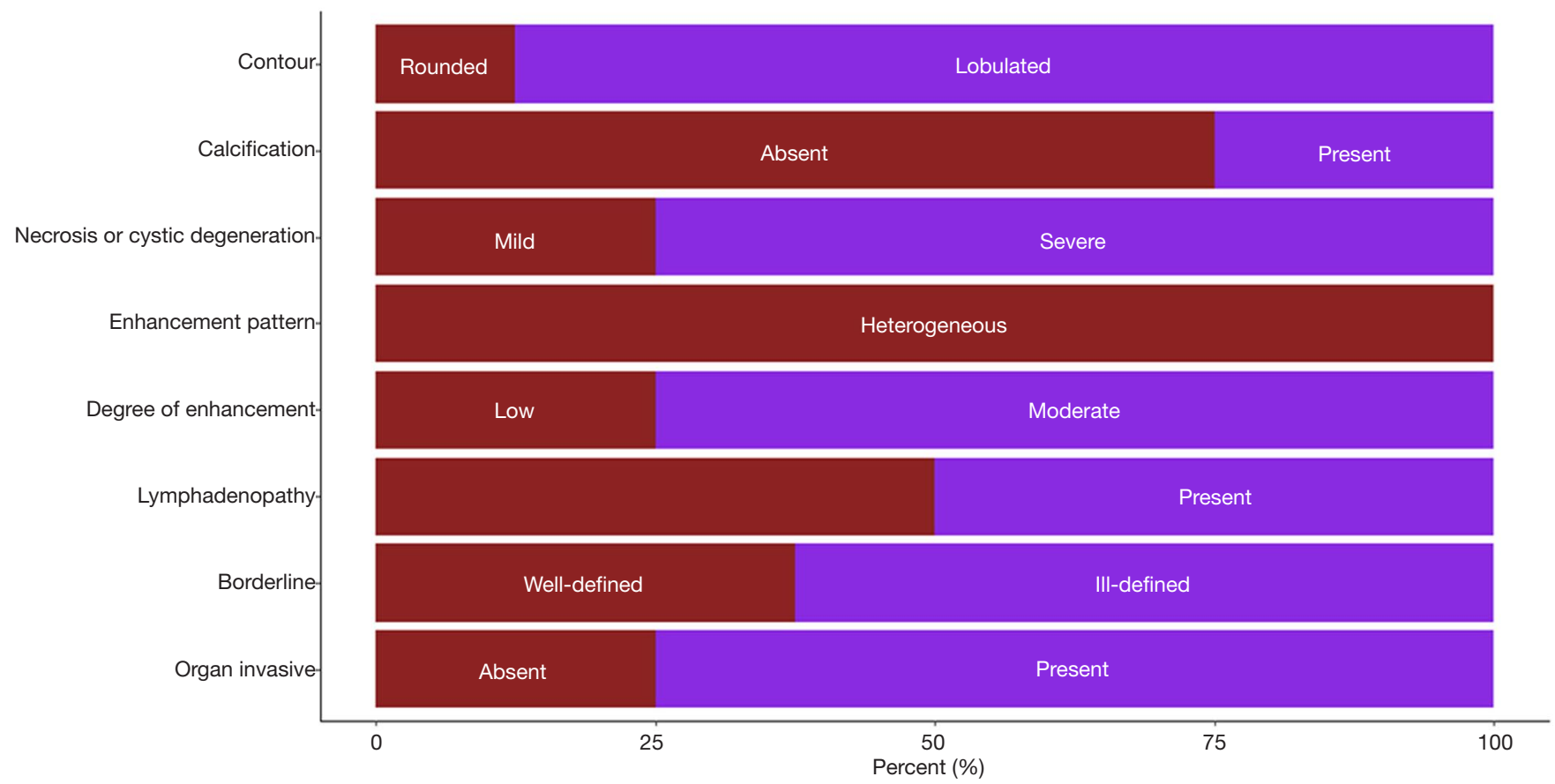

Figure 2 The incidences of tumor imaging features were visually described using a stacked bar plot. All the variables were dichotomous.
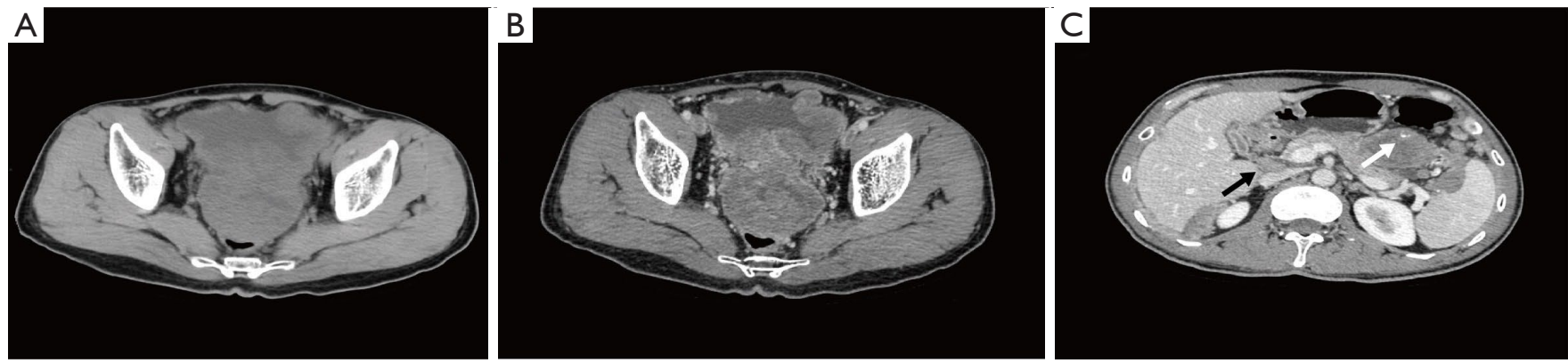

Figure $3 \mathrm{EES}$ in the pelvis. (A) Unenhanced CT image shows a large ill-defined mass in the pelvis involving the bladder and oppressing the rectum. (B) Contrast-enhanced CT image shows mild heterogeneous enhancement caused by unenhanced areas indicating the necrosis or cystic degeneration. (C) Contrast-enhanced CT image in upper slice shows multiple metastatic lesions in enterocoelia and peritoneum. The lymphadenopathy can be seen in the hepatic hilar area (black arrow) and some punctate calcifications (white arrow) exist in the metastatic lesion.

excess of $65 \%$ (7). However, in our study, the OS of EES was about $20 \%$ at two-year follow-up. The therapeutic strategies for EES are based on the experience garnered from the treatment of SES. Several studies have highlighted that the neoadjuvant chemotherapy was a standard therapeutic approach for localised EES $(7,8)$. However, only $15 \%(3 / 20)$ of patients in the current study underwent surgery and agreed to neoadjuvant chemotherapy. Meanwhile, radiotherapy is recommended for patients with inadequate surgical margins (23). Nonetheless, only two patients $(2 / 4,50 \%)$ with suboptimal margins agreed to postoperative radiotherapy in the current study. Moreover, VAC alternating with IE, which is the normative aggressive chemotherapy protocol (24), was used in less than half of our patients. Any or all of these reasons could explain why patients with localised EES in our study had poor prognosis that did not differ statistically from the prognosis of patients with metastatic EES. At present, the widely 

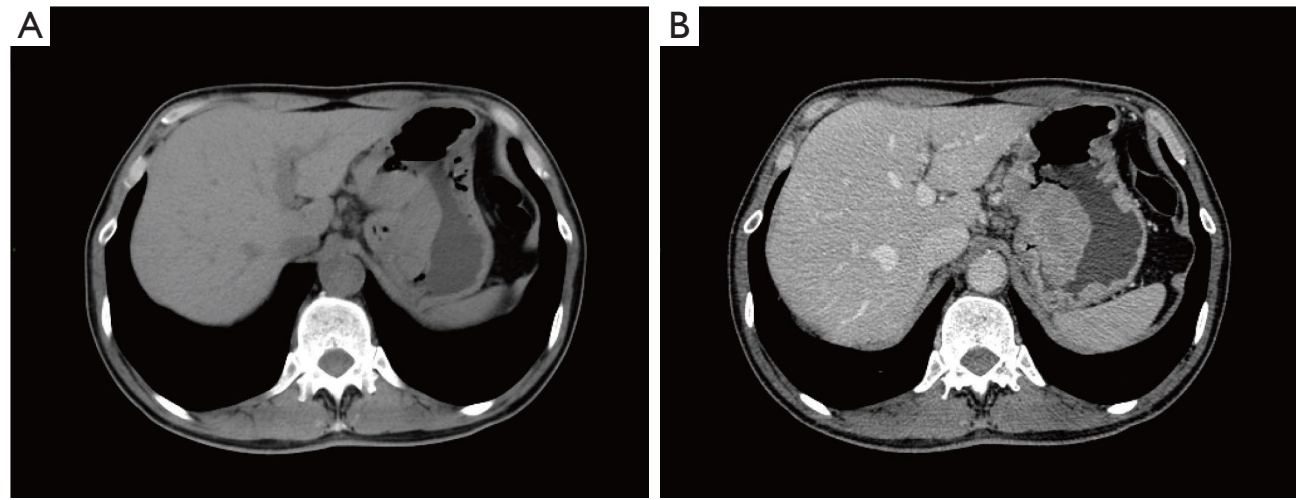

Figure 4 EES in the stomach. (A) Unenhanced CT image shows an irregular thickening with ill-defined boundary in the lesser curvature. (B) Contrast-enhanced CT image shows mild heterogeneous enhancement in the interior of the tumour.
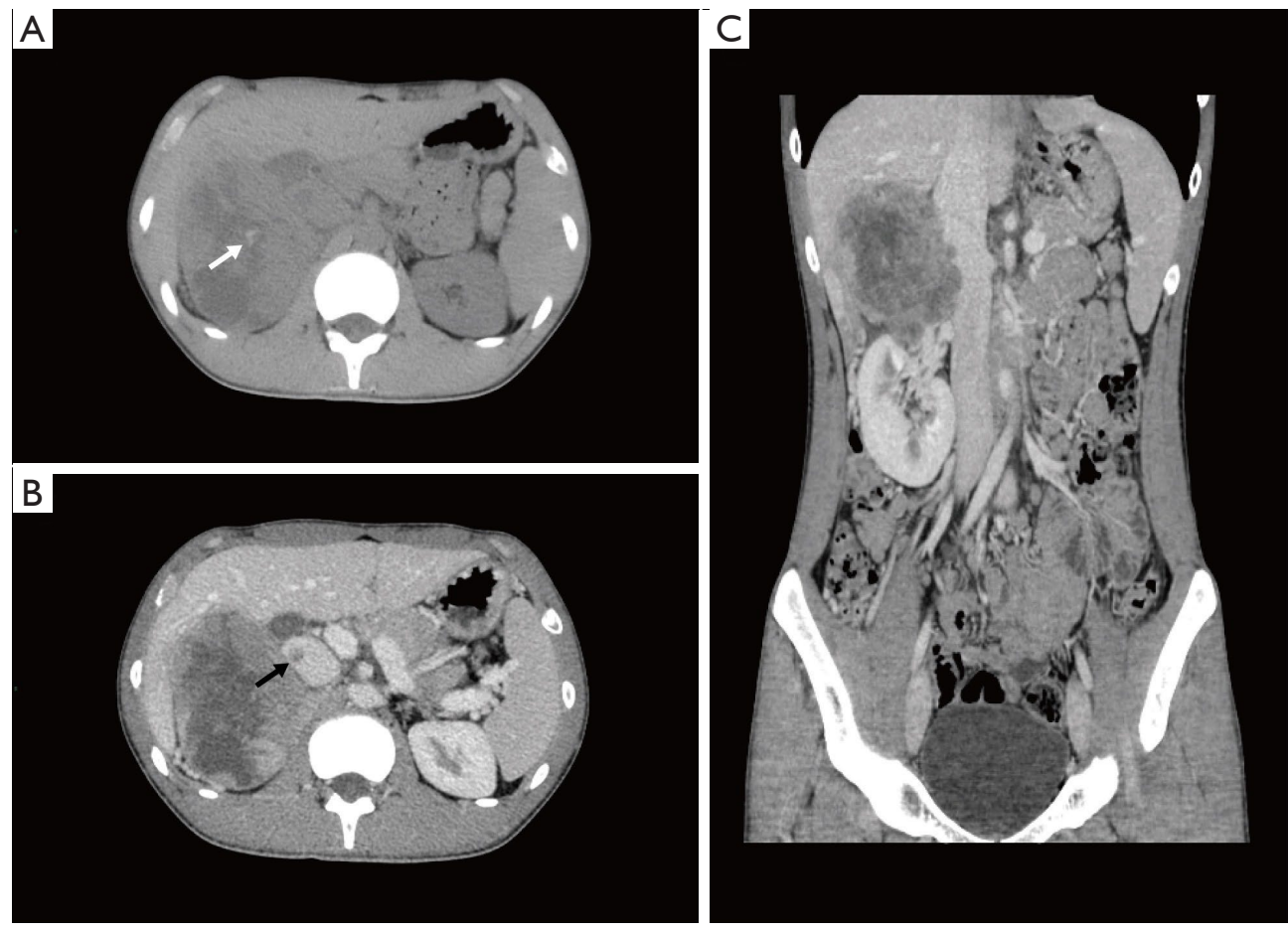

Figure 5 EES in the adrenal gland. (A) Unenhanced CT image shows that a huge lesion with multiple low density areas and lamellar calcification (white arrow) compresses the right lobe of liver. (B) Contrast-enhanced CT image shows heterogeneous enhancement because of the necrosis or cystic degeneration and a filling defect in the postcava indicating the existing of tumour thrombosis (black arrow). (C) Coronal scan CT image shows that tumour displaces the normal adrenal gland and involves the postcava.

accepted view is that aggressive multimodality treatment should be performed in EES. That is, treatment should begin with neoadjuvant chemotherapy, followed by local treatment including surgery and radiotherapy, and finally, multiagent adjuvant chemotherapy (13). Research suggests that regional lymph node metastasis is associated with disseminated disease and poor prognosis (25). Applebaum et al. concluded that lymph node metastasis was common in extraskeletal ESFTs (26). Our study also found that lymph node involvement was an independent risk factor for poor prognosis. This finding has highlighted the importance of evaluating the status of regional lymph nodes when 
assessing EES. As such, technologies such as sentinel lymph node biopsies and routine regional lymph node dissection have potential application prospects for the treatment of EES.

The basic imaging features of EES, especially for endoceliac EES with insidious onset and poor prognosis, remain unclear. The application of CT can provide as much imaging information about ESFTs as MRI, is cheaper and more convenient than MRI in clinical practice (20). Endoceliac EES mimics other malignant lesions at corresponding sites, such as carcinoma of the kidney, prostate, colorectum, ovary, and so on. Therefore, it is crucial that we have a thorough general understanding of the CT features of endoceliac EES, as these patients tend to present to a wide range of departments, including general surgery, gynaecology, and urinary surgery departments; this would ensure that the various specialists who encounter these patients are aware of the importance of standard treatment for EES. Commonly, EES is a highgrade malignant tumour that tends to be large in size and has a quick growth rate. It is also commonly accompanied by a lobulated contour and necrosis, which can lead to a heterogeneous enhancement pattern on CT examination (1,6,20,27-29). An imbalance associated with the tumour's fast growth rate and insufficient blood supply could be why these tumours tend to exhibit severe necrosis. Second, almost all publications on EES report ill-defined borderlines $(29,30)$ and endoceliac lesions, like abdominal EES, more commonly exhibit organ invasion on CT, as compared to peripheral lesions (27). Finally, EES often presents with a moderate degree of enhancement (28) and absence of calcification $(7,20,27)$. On the other hand, the current study also encountered some extraordinary findings related to the imaging features of endoceliac EES. The majority of imaging studies have indicated that lymphadenopathy is a rare CT feature in EES (7,27-29). However, half of the patients with endoceliac EES in this study presented with lymphadenopathy. Together with the findings of previous clinical analyses, the current findings suggest that serious preoperative evaluation, careful intraoperative management, and rigorous postoperative staging of regional lymph nodes are vital in the treatment of endoceliac lesions.

Our study also had several limitations. First, the main weakness of this study was the small sample size. This could be why endoceliac EES only trended towards poorer OS than surface, though this was not statistically significant. Second, our institution is famous for its urology and general surgery departments; thus, there was referral bias, and as such, this study had a large portion of patients with EES originating from the urinary and digestive systems. Third, despite the long-time span of this study, the follow-up periods for some patients were less than three years. In addition, the chemotherapy protocols varied across patients due to the time span of the study. Finally, this study was a retrospective study with inevitable selective bias. In spite of its limitations, this study is the largest published series of EES patients in China. The data acquired from reviewing clinicopathological and imaging data has potential value for improving preoperative diagnostic accuracy and formulating a standard treatment strategy for this rare disease.

In conclusion, large tumour size, non-surgical treatment, and regional lymph nodes metastasis were risk factors for poor prognosis in EES. In addition, regional lymph nodes metastasis was an independent predictor of OS in EES patients who underwent surgery. Endoceliac EES, which tends to be misdiagnosed preoperatively, exhibited the following common CT features: lobulated contour, absence of calcification, severe necrosis, moderate heterogeneous enhancement, ill-defined borderline, and organ invasion. Lymphadenopathy was also relatively common. Thus, the findings of this study contribute to the understanding of both the clinicopathological and imaging characteristics of this rare disease.

\section{Acknowledgments}

Funding: None.

\section{Footnote}

Reporting Checklist: The authors have completed the STROBE reporting checklist. Available at https://dx.doi. org/10.21037/tcr-21-607

Data Sharing Statement: Available at https://dx.doi. org/10.21037/tcr-21-607

Peer Review File: Available at https://dx.doi.org/10.21037/ tcr-21-607

Conflicts of Interest: The authors have completed the ICMJE uniform disclosure form (available at https://dx.doi. org/10.21037/tcr-21-607). The authors have no conflicts of interest to declare.

Ethical Statement: The authors are accountable for all 
aspects of the work in ensuring that questions related to the accuracy or integrity of any part of the work are appropriately investigated and resolved. The study was conducted in accordance with the Declaration of Helsinki (as revised in 2013). The retrospective study was approved by the Institutional Ethics Committee of Peking University First Hospital (No. 2021-365) and the verbal informed consent was obtained from all the patients.

Open Access Statement: This is an Open Access article distributed in accordance with the Creative Commons Attribution-NonCommercial-NoDerivs 4.0 International License (CC BY-NC-ND 4.0), which permits the noncommercial replication and distribution of the article with the strict proviso that no changes or edits are made and the original work is properly cited (including links to both the formal publication through the relevant DOI and the license). See: https://creativecommons.org/licenses/by-nc-nd/4.0/.

\section{References}

1. El Weshi A, Allam A, Ajarim D, et al. Extraskeletal Ewing's sarcoma family of tumours in adults: analysis of 57 patients from a single institution. Clin Oncol (R Coll Radiol) 2010;22:374-81.

2. Ambros IM, Ambros PF, Strehl S, et al. MIC2 is a specific marker for Ewing's sarcoma and peripheral primitive neuroectodermal tumors. Evidence for a common histogenesis of Ewing's sarcoma and peripheral primitive neuroectodermal tumors from MIC2 expression and specific chromosome aberration. Cancer 1991;67:1886-93.

3. Xie CF, Liu MZ, Xi M. Extraskeletal Ewing's sarcoma: a report of 18 cases and literature review. Chin J Cancer 2010;29:420-4.

4. Delattre O, Zucman J, Melot T, et al. The Ewing family of tumors--a subgroup of small-round-cell tumors defined by specific chimeric transcripts. N Engl J Med 1994;331:294-9.

5. Tsokos M, Alaggio RD, Dehner LP, et al. Ewing sarcoma/ peripheral primitive neuroectodermal tumor and related tumors. Pediatr Dev Pathol 2012;15:108-26.

6. Huh J, Kim KW, Park SJ, et al. Imaging Features of Primary Tumors and Metastatic Patterns of the Extraskeletal Ewing Sarcoma Family of Tumors in Adults: A 17-Year Experience at a Single Institution. Korean J Radiol 2015;16:783-90.

7. Javery O, Krajewski K, O'Regan K, et al. A to Z of extraskeletal Ewing sarcoma family of tumors in adults: imaging features of primary disease, metastatic patterns, and treatment responses. AJR Am J Roentgenol 2011;197:W1015-22.

8. Maki RG. Pediatric sarcomas occurring in adults. J Surg Oncol 2008;97:360-8.

9. Balamuth NJ, Womer RB. Ewing's sarcoma. Lancet Oncol 2010;11:184-92.

10. Yock TI, Krailo M, Fryer CJ, et al. Local control in pelvic Ewing sarcoma: analysis from INT-0091--a report from the Children's Oncology Group. J Clin Oncol 2006;24:3838-43.

11. Takenaka S, Naka N, Obata H, et al. Treatment outcomes of Japanese patients with Ewing sarcoma: differences between skeletal and extraskeletal Ewing sarcoma. Jpn J Clin Oncol 2016;46:522-8.

12. Lynch AD, Gani F, Meyer CF, et al. Extraskeletal versus Skeletal Ewing Sarcoma in the adult population: Controversies in care. Surg Oncol 2018;27:373-9.

13. Applebaum MA, Worch J, Matthay KK, et al. Clinical features and outcomes in patients with extraskeletal Ewing sarcoma. Cancer 2011;117:3027-32.

14. Krakorova DA, Kubackova K, Dusek L, et al. Advantages in Prognosis of Adult Patients with Ewing Sarcoma: 11-years Experiences and Current Treatment Management. Pathol Oncol Res 2018;24:623-30.

15. Chen Z, Yang J, Sun J, et al. Gastric gastrointestinal stromal tumours $(2-5 \mathrm{~cm})$ : Correlation of CT features with malignancy and differential diagnosis. Eur J Radiol 2020;123:108783.

16. Jiang S, Wang G, Chen J, et al. Comparison of clinical features and outcomes in patients with extraskeletal vs skeletal Ewing sarcoma: an SEER database analysis of 3,178 cases. Cancer Manag Res 2018;10:6227-36.

17. Qureshi SS, Laskar S, Kembhavi S, et al. Extraskeletal Ewing sarcoma in children and adolescents: impact of narrow but negative surgical margin. Pediatr Surg Int 2013;29:1303-9.

18. Raney RB, Asmar L, Newton WA Jr, et al. Ewing's sarcoma of soft tissues in childhood: a report from the Intergroup Rhabdomyosarcoma Study, 1972 to 1991. J Clin Oncol 1997;15:574-82.

19. Omori $M$, Ueno $M$, Ogawa $M$, et al. Primary cutaneous extraskeletal Ewing's sarcoma/PNET: possibility of better prognosis than deep ES/PNET. Eur J Dermatol 2014;24:126-7.

20. Dick EA, McHugh K, Kimber C, et al. Imaging of noncentral nervous system primitive neuroectodermal tumours: diagnostic features and correlation with outcome. 
Clin Radiol 2001;56:206-15.

21. Cash T, McIlvaine E, Krailo MD, et al. Comparison of clinical features and outcomes in patients with extraskeletal versus skeletal localized Ewing sarcoma: A report from the Children's Oncology Group. Pediatr Blood Cancer 2016;63:1771-9.

22. Castex MP, Rubie H, Stevens MC, et al. Extraosseous localized ewing tumors: improved outcome with anthracyclines--the French society of pediatric oncology and international society of pediatric oncology. J Clin Oncol 2007;25:1176-82.

23. Cotterill SJ, Ahrens S, Paulussen M, et al. Prognostic factors in Ewing's tumor of bone: analysis of 975 patients from the European Intergroup Cooperative Ewing's Sarcoma Study Group. J Clin Oncol 2000;18:3108-14.

24. Grier HE, Krailo MD, Tarbell NJ, et al. Addition of ifosfamide and etoposide to standard chemotherapy for Ewing's sarcoma and primitive neuroectodermal tumor of bone. N Engl J Med 2003;348:694-701.

25. Bernstein ML, Devidas M, Lafreniere D, et al. Intensive therapy with growth factor support for patients with Ewing tumor metastatic at diagnosis: Pediatric Oncology
Group/Children's Cancer Group Phase II Study 9457--a report from the Children's Oncology Group. J Clin Oncol 2006;24:152-9.

26. Applebaum MA, Goldsby R, Neuhaus J, et al. Clinical features and outcomes in patients with Ewing sarcoma and regional lymph node involvement. Pediatr Blood Cancer 2012;59:617-20.

27. Somarouthu BS, Shinagare AB, Rosenthal MH, et al. Multimodality imaging features, metastatic pattern and clinical outcome in adult extraskeletal Ewing sarcoma: experience in 26 patients. Br J Radiol 2014;87:20140123.

28. Gupta P, Hari S, Thulkar S. Imaging spectrum of peripheral primitive neuroectodermal tumours. Singapore Med J 2013;54:463-2.

29. Li X, Zhang W, Song T, et al. Primitive neuroectodermal tumor arising in the abdominopelvic region: CT features and pathology characteristics. Abdom Imaging 2011;36:590-5.

30. Kim MS, Kim B, Park CS, et al. Radiologic findings of peripheral primitive neuroectodermal tumor arising in the retroperitoneum. AJR Am J Roentgenol 2006;186:1125-32.
Cite this article as: Chen Z, Jiao Y, Liu Z, Yang J, Sun J, Wang P. Extraskeletal Ewing's sarcoma: outcomes and CT features of endoceliac lesions. Transl Cancer Res 2021;10(9):4065-4075. doi: $10.21037 /$ tcr-21-607 